\title{
LA HISTORIA DE LA CIENCIA COMO HERRAMIENTA PARA LA CONSTRUCCIÓN DE SIGNIFICADOS EN LOS CURSOS DE FÍSICA UNIVERSITARIOS: UN EJEMPLO EN FUERZA Y MOVIMIENTO
}

\author{
Diana Patricia Rodríguez Pineda,bogota65 @ hotmail.com* \\ José González Florez, gonzalej @ uni.pedagogica.edu co**
}

\begin{abstract}
This article shows a classroom physics research from a historical point of view and it compares student explanations with those explanations which have been given in the past. The classroom work takes place around four problematical situations in mechanics.
\end{abstract}

\section{RESUMEN}

El artículo muestra una investigación de aula en física introductoria, desde una perspectiva histórica y compara las explicaciones de los alumnos con las que se han dado a lo largo de la historia. El trabajo de aula se desarrolla en torno a cuatro situaciones problemáticas de la mecánica.

Palabras claves: Enfoque Histórico, Constructivismo, Ideas Previas, Cambio Conceptual, Didáctica, Física Escolar, Aristóteles, Galileo, Newton.

\section{INTRODUCCIÓN}

Los elementos conceptuales que se exponen en el presente texto se elaboran a través de cuatro grandes apartados: En la primera parte se plantea eL problema de investigación y se asume como hipótesis de trabajo la siguiente: Si se orienta un curso de física desde una perspectiva histórica, pretendiendo que los alumnos identifiquen sus ideas explicativas con las que han estado presentes a lo largo de la historia de la física y que también identifiquen por qué cambiaron esos paradigmas, se logrará igualmente en alguna medida una transformación o cambio conceptual de los alumnos. En la segunda parte se exponen los referentes teóricos que se utilizaron como ejes articuladores del proceso investigativo. En la tercera parte se da cuenta del trabajo decampo mediante un breve análisis en torno a cuatro situaciones problemáticas especificas: un mundo geocéntrico dentro de un universo heliocéntrico; concepción indiferenciada de gravedad, fuerza gravitacional y peso; de la fuerza a la fuerza neta y del término de fuerza. Y finalmente se presentan las conclusiones y sugerencias, producto del trabajo de investigación

Es pertinente aclarar que con el en-foque didáctico propuesto en este trabajo se pretende mostrar la ciencia como un actividad cultural, construida por un grupo social determinado, de esta manera se desea evitar el carácter de exterioridad como suele tratarse la ciencia, vista como productos elaborados por personas ajenas. Igualmente al comparar las explicaciones dadas en el aula de clase y las de los científicos reconocidos

\footnotetext{
* Profesora del Departamento de estudios Educativos de la Facultad de Artes y Humanidades, Universidad de Caldas, Manizales.

Profesor del Departamento de Física, Universidad Pedagógica Nacional
} 
se quiere pues acercar a los estudiantes al "quehacer científico" y ver las relaciones entre el pensamiento común y el de la ciencia.

\section{PRIMERA PARTE: Antecedentes}

Un marco de investigación comprende, para empezar compromisos de tipo pragmático: ¿cuál es el interés en construir o aportar elementos de tipo teórico o empírico a determinado trabajo?. es decir, cuál es el problema a resolver ya qué campo de fenómenos se pretende aplicar?, ¿cuál es la hipótesis que se propone como solución al problema? En este contexto general de exposición de motivos, se desarrollan a continuación dos cuestiones: Planteamiento del Problema, y objetivos.

\section{A. Planteamiento del problema}

La orientación tradicional de La enseñanza de las ciencias está centrada fundamentalmente en los contenidos (Hammer, 1994); contenidos que se fundamentan en fórmulas para la solución de ejercicios y problemas. que resultan poco significativos, de esta manera los maestros no permiten, ni propician que se de la toma de consciencia como fase fundamental del aprendizaje; de tal suerte que los alumnos siguen explicando los fenómenos físicos con sus mismas ideas, generando así dos esferas inconexas de conocimiento, que desde la perspectiva epistemológica Kuhniana se pueden denotar, de acuerdo con Susan (Carey, 1992), como dos marcos inconmesurables de conocimiento, uno para el contexto de su vida cotidiana y otro para el contexto escolar.

Entonces desde lo didáctico se hace necesario proponer un curso de física para estudiantes de primer semestre con una metodología no tradicional —en la cual el maestro dicta' su clase y los alumnos demuestran lo que 'aprendieron' a través de 3 o 4 parciales-; un curso que permita un espacio de reflexión y análisis y el reconocimiento por parte tanto del maestro como de los mismos alumnos sobre sus esquemas explicativos alternativos al denominado conocimiento científico, y sobre cómo sus preconceptos están muy cercanos a la teoría aristotélica y a la del ímpetu.

Se desconoce, como lo afirman (Osborne y Witrock, 1983) que los alumnos desarrollan ideas sobre su mundo, construyen significados para las palabras que se usan en ciencia y despliegan estrategias para conseguir explicaciones sobre cómo y por qué las cosas se comportan como lo hacen, además sus ideas previas sobre los fenómenos físicos son por lo general diferentes a las científicas y no son modificadas por la acción escolar (Champagne, Gustone, Klopfer, 1985; Hewson 1990).

El otro es el hecho de que un alto porcentaje de maestros de física, no conoce el desarrollo histórico de la disciplina que orientan, ni el devenir de la ciencia misma, pues la gran mayoría de veces no han sido formados en esta disciplina, sino en áreas afines y por diferentes circunstancias, se ven abocados a orientar los cursos de física. Esta y otras razones pueden llevar al profesorado a desconocer el paralelismo que existe entre algunas concepciones de los alumnos y determinadas teorías pasadas en la historia de las ciencias (Clement, 1983; Whitaker, 1983; Pozo, 1987; Fischbein et al., 1989; Mathews, 1990), como en el caso de sus ideas previas sobre movimiento y fuerza con la teoría aristotélica y la teoría medieval del ímpetus (McCloskey, 1983); concepciones que no han sido fáciles de cambiar y que al igual que la modificación de los puntos de vista de los estudiantes requieren cierto tiempo, conocimiento que podría ser de gran utilidad en el trabajo didáctico de los docentes. 
El cuadro vislumbrado hasta aquí, nos da elementos para reflexionar sobre La existencia real en el contexto propio del proceso enseñanza-aprendizaje de la relación entre las ideas previas (aLumnos), la historicidad de la disciplina (saber específico) y la didáctica (profesor).

Lo anterior nos llevó a plantear las siguientes preguntas de investigación:

- ¿Existe en el contexto propio del aprendizaje algún tipo de paralelismo entre las ideas de los alumnos y las teorías que en algún momento fueron válidas en la historia de la física?

- ¿Ayuda al cambio de paradigma explicativo de los fenómenos físicos, el hecho de que ellos identifiquen desde la historia las inconsistencia de sus marcos explicativos?

- ¿La didáctica empleada por el profesor, posibilita que el aprendizaje sea realmente significativo para el estudiante?

- ¿Cómo pueden nuestros estudiantes, hacer uso actualmente de la historia de la física que se les presenta?

Con base en lo anterior asumiremos como hipótesis de trabajo la siguiente:

Sise orienta un curso de física desde una perspectiva histórica, pretendiendo que los alumnos identifiquen sus ideas explicativas, con las que han estado presentes a lo largo de la historia de la física y que así mismo identifiquen por qué cambia ron esos paradigmas, se logrará también en alguna medida una transformación conceptual en los alumnos.

\section{B. Objetivos}

- Lograr que los alumnos identifiquen sus ideas explicativas, con las que han estado presentes a lo largo de la historia de la física y que también identifiquen por qué cambiaron esos paradigmas.

- Analizar el efecto didáctico, en el aula de clase, que tiene el enfoque histórico respecto al Cambio Conceptual, en particular el cambio de paradigma de Aristóteles al de Newton.

- Aportar algunos elementos de análisis y reflexión, en torno a la pertinencia, a la relevancia y a los efectos de un curso de física en torno al tema de fuerza y movimiento.

\section{SEGUNDA PARTE. Ejes teóricos articuladores}

\section{A. La alternativa constructivista para la enseñanza de las ciencias}

El constructivismo constituye, antes que nada, una posición epistemológica, es decir, referente a cómo se origina, estructura, valida y cambia el conocimiento. Esta postura epistemológica tiene sus fundamentos en el relativismo y en el contextualismo (Kuhn, Feyerabend, Toulmin y Laudan); surge como una alternativa a las clásicas posiciones epistemológicas, especialmente al empirismo y al racionalismo, que a pesar de sus fuertes diferencias, coinciden con base en lo planteado por (Pérez Ransanz, 1999), en 4 postulados básicos, a saber: establecer la existencia de un único método universal que corresponde a las reglas metodológicas que garantizan la correcta práctica científica y el 
auténtico conocimiento; la necesidad de un criterio de validez universal; la existencia de relaciones lógicas que conectan las hipótesis con la evidencia; y el progresivo desarrollo del conocimiento científico, en el sentido de que se tiende a la teoría correcta del mundo.

Particularmente, sobre el punto de vista constructivista del aprendizaje, vale la pena destacar que éste se centra en la importancia del significado construido por las personas en sus intentos de dar sentido al mundo.

Así pues, desde esta concepción es necesario tomar en consideración las ideas previas, el desarrollo y cambio conceptual y la estructuración del conocimiento de los estudiantes. Además, implica hacer ver a la enseñanza como algo dinámico (transformación conceptual) y en proceso de construcción (similar a la estructuración conceptual alcanzada en la historia de la ciencia); y también tiene como consecuencia que las entidades conceptuales se presenten como elementos de enseñanza sujetos a interpretación, para lo cual se requiere de formas de representación que les den significado.

Las Ideas Previas. Gran parte de las investigaciones sobre el aprendizaje en el campo de la educación en ciencias experimentales, especialmente en las últimas dos décadas, ha constituido, en sentido Lakatosiano, un programa de investigación sobre las ideas previas de los alumnos, especialmente a partir de los trabajos experimentales realizados por (Viennot, 1979). En la actualidad, la literatura referente a las ideas intuitivas de los alumnos acerca de la ciencia esta creciendo significativamente (Base Duit, 1994 y los Handbook, 1994 y 1998); al respecto (Furió y Guisasola, 1999) destacan que la revista española Enseñanza de las Ciencias le ha dedicado entre los años 1983 y 1992, el 38\% del total de sus artículos publicados a este tema de las ideas previas. Por lo que hoy día está generalmente aceptado que los niños desarrollan ideas y creencias sobre el mundo natural mucho antes de que entren en el sistema escolar formal, y se reconoce por muchos investigadores la importancia de estas concepciones en la enseñanza.

Existe una gran variedad terminológica en la literatura para referirse a los puntos de vista o ideas que tienen los alumnos, por ejemplo: ideas previas, preconceptos, conceptos equivocados, esquemas alternativos, teorías de los niños, concepciones alternativas, etc. Cada término depende de la mirada del investigador, pues a cada denominación subyace una concepción epistemológica y psicológica distinta; así pues, quienes utilizan el término 'misconceptions'o 'errores conceptuales', consideran que las ideas de los alumnos son siempre erróneas, lo que implica una visión peyorativa del complejo proceso conceptual que implica construir nociones o concepciones. Otros (Driver y Easley, 1978) han dado el nombre de 'marcos de funcionamiento alternativos' a las conceptualizaciones personales de los escolares sobre la ciencia, por considerarlas como ideas coherentes del mundo basadas en sus propias experiencias; como hipótesis particulares y especulaciones referentes a eventos, cargadas de significado.

\section{B. La historicidad en la enseñanza de las ciencias}

La aproximación histórica parece tener un contenido muy interesante para el estudiante, porque le muestra la posibilidad de una discusión critica sobre el papel y el poder de la ciencia, relacionándola además, con otras actividades humanas. Además, el conocimiento de la historia de su disciplina es esencial para el profesor, puesto que lo provee de elementos para detectar los posibles obstáculos que enfrentan los estudiantes en el proceso de aprendizaje, es más, sirve al docente para generar preguntas conflictivas. 
Nosotros compartimos la hipótesis de que, detrás de todos estos argumentos que justifican el uso de la historia de las ciencias en la enseñanza y en la formación de profesores de ciencias, existe un argumento más simple, más fácilmente observado en la vida diaria y menos pretencioso, que es fundamental: usar la historia del conocimiento científico es, por encima de todo, tratar de transformar el frío, disertativo, e impersonal lenguaje científico (cuando este es presentado como un producto terminado) en un narrativo, descriptivo y secuencial lenguaje, capaz de interrelacionar lo que había sido oscuro, y por ende mucho más cercano a las habilidades cognitivas de los estudiantes.

Quienes nos interesamos en las respuestas de los estudiantes a la hora dar cuenta de un problema de Física o explicar un fenómeno, e incluso en varias de las investigaciones sobre este tema, tendemos a rotularlas, calificando las concepciones de los estudiantes sobre la fuerza y el movimiento como aristotélicas; otros manifiestan que son semejantes a la de la teoría medieval del ímpetu. En cierto sentido podría decirse que estas etiquetas son irrelevantes, dado que ningún niño tiene concepciones acerca de estas cuestiones de la amplitud y de la consistencia interna de, ARISTÓTELES, o del estudioso francés del siglo XIV BURIDAN. No obstante, estas etiquetas sirven para recordarnos que tanto la perspectiva aristotélica y la del ímpetu acerca de la fuerza y al movimiento fueron consideradas lógicas y razonables por numerosos eruditos por más de 2000 anos.

Evidentemente una razón de peso para ello es que resulta muy posible hacer observaciones cotidianas de los fenómenos que apoyan ambas visiones. En general, es muy razonable pensar que gran cantidad de nuestros estudiantes, y de nosotros mismos -en algún momento--hemos utilizado tales observaciones para construir los modelos que nos permiten dar sentido a nuestro mundo. Estos modelos, si bien en algunos casos están mal articulados y son inapropiados según la perspectiva de la física, están presentes en el aula.

Para lograr el aprendizaje es necesario partir del modelo conceptual previo a la ciencia escolar, con el fin de lograr un cambio conceptual y construir dicho aprendizaje. Es aquí donde la historia de las ciencias (y de la física en particular) juegan un papel importante en la didáctica de la física, puesto que por un lado existe similitud entre las ideas previas de los alumnos, con teorías que en otra época fueron válidas.

Fuerza y Movimiento en la Física Escolar. Uno de los criterios para delimitar el campo de acción del trabajo fue el dar prioridad al tema que dentro del campo conceptual de la física, constituye las bases de la construcción del conocimiento físico, por lo cual decidimos trabajar sobre movimiento y fuerza. Al abordar el estudio de la física es fundamental hablar sobre el movimiento, este concepto tan ligado a las ideas de materia, espacio y tiempo, y por ello a la naturaleza misma del universo, y ese nos conlleva intuitivamente a hablar del concepto de fuerza. Estos temas son centrales en el desarrollo completo de la física, máxime si con quienes se va a trabajar son estudiantes que por primera vez se encuentran formalmente en el mundo de la física, en la Universidad.

Además, este tema está reportado en la literatura como uno de los más resistentes al cambio conceptual, muestra de ello es el alto número de investigaciones que se refieren a la identificación de las ideas previas de los estudiantes. Así por ejemplo, la revisión bibliográfica sobre concepciones alternativas realizada por (Pfundt y Duit, 1998), señalaban más de 4000 referencias, en el Handbook of Research on Science Teaching and Learning, (Wandersee, 1994) menciona 700 estudios sobre física de los cuales 300 de ellos son sobre dinámica; también en un análisis (Solano, Jiménez, y Marín, 2000), sobre "la metodología utilizada en las investigaciones que se refieren a lo que el alumno 
sabe sobre fuerza', reseñan 29 trabajos relacionados con el tema, pero todos ellos de carácter eminentemente descriptivo.

Vale la pena resaltar, que la formulación de Newton y sus leyes, constituyen la baso de la 'física escolar'. Dentro de la perspectiva de los cursos tradicionales de física (de mecánica en particular) aún los universitarios, la 'fórmula' que más frecuentemente tienen que usar los alumnos es $\mathrm{F}=$ ma (Fuerza $=$ masa $\mathrm{x}$ aceleración). Sin embargo, las sutilezas que subyacen a la misma se ponen de manifiesto raras veces. Las leyes requieren el reconocimiento de las tuerzas, es decir, sólo sí se distinguen claramente las fuerzas individuales que actúan sobre un cuerno, pueden llegar a tener sentido dichas leyes. Además, la comprensión de $\mathrm{F}=$ ma es mucho más difícil porque a menudo choca con las concepciones intuitivas de los alumnos sobre el movimiento.

Por tanto, a diferencia de la teoría de Aristóteles, en la que la velocidad constante y uniforme es natural no necesita explicación. En la de Newton, el cambio producido en el movimiento, o aceleración, si necesita ser explicado y esa explicación se basa en fuerzas resultantes. La segunda ley específica la relación; mientras antes la fuerza y la velocidad se consideraban proporcionales, según las leyes de Newton lo son la fuerza y la aceleración.

\section{TERCERA PARTE: Descripción y análisis}

\section{A. Descripción}

La investigación es de tipo etnográfica

- cualitativa, basada en el análisis de una experiencia particular, en la cual se utilizaron actividades de dos tipos: actividades de reconstrucción racional y actividades de tipo dialógico.

Para las actividades del primer tipo utilizamos como canal fundamental la lectora-con sus respectivas revisiones-, la cual daba pie a las actividades del segundo tipo, que buscaban una interacción entre los procesos de construcción de los estudiantes y los de los científicos.

Para las actividades de reconstrucción racional usamos un texto con información histórica (Hetch) y videos de la serie cosmos (Carl Sagan), al igual que algunas consultas bibliográficas y para las de tipo dialógico, realizamos sesiones de discusión y socialización.

Recolección de Datos. Durante el desarrollo del proyecto se emplearon esencialmente tres técnicas que permitieron obtener datos confiables y concretos, las cuales contribuyeron con el seguimiento continuo a todo el proceso, estas son:

La observación. Permanentemente se utilizó esta herramienta para obtener información de las diferentes respuestas y cuestionamientos que los mismos estudiantes se hacían sobre las lecturas y las discusiones por grupos de trabajo, registrando expresiones como: Aún estamos en el pensamiento de hace 2000 años.

Entrevistas. Al final del curso se realizó un examen oral, a manera de entrevista, con cada uno de los alumnos del grupo, en la cual se trataron primordialmente las situaciones 
problémicas, de las que se dará cuenta más adelante en la sección de las preguntas o ejemplos.

\section{Cuestionarios}

Durante el curso se solicitó a los alumnos que respondieran algunos cuestionarios algunos a manera de pequeñas evaluaciones, como parte del curso-, antes y/o después de cada sección temática, para identificar sus preconceptos y para tratar de identificar si éstos cambian o no.

Dos meses después de terminado el curso, con el fin de corroborar la información y algunos aspectos relacionados con la situación y el medio donde se llevo a acabo la investigación, pedimos a un pequeño grupo de alumnos que por escrito dieran cuenta de la metodología del curso; es decir, de alguna manera so les solicitó que lo evaluaran y que reseñara los conceptos que habían sino mas significativos para ellos a lo largo del mismo. La muestra de alumnos fue seleccionada al azar.

Selección de Participantes y su Situación. Las clases de las cuales se tomo la información que aquí presentamos, fue ron parte de un curso de introducción a la Física, de un grupo de 32 alumnos del primer semestre de la Licenciatura en Biología y Química de la Universidad de Caldas, quienes a lo largo de 4 meses trabajaron con la metodología anteriormente planteada para el curso.

Vale la pena resaltar que un gran porcentaje (62.5\%) de ellos ingreso a esta carrera por segunda opción, es decir se presentaron a otras carreras como Medicina, Veterinaria y Enfermería, pero al no alcanzar cupo en ellas, fueron aceptados en ésta licenciatura; lo cual de antemano genera un contexto de investigación muy particular, pues no solo debíamos dar cuenta de los objetivos propuestos inicialmente, sino que adicionalmente a través de la hipótesis de trabajo propuesta debíamos motivar a nuestros estudiantes, quienes aspiraban a presentarse nuevamente 110 a su primera opción y al principio del de semestre solo les parecían importantes las materias más afines entre las dos carreras, es decir las asignaturas de la rica de biológicas y de química.

Así, que no solo estaba el reto en la construcción de significados a partir de la aproximación histórica de los conceptos de fuerza y movimiento, sirio que también había que lograr que los alumnos se interesaran por la clase en si.

Los estudiantes provenían en su gran mayoría de la ciudad de Manizales y de colegios públicos, el resto eran de provincia y unos cuentos eran originarios de Pasto y Cali. Casi todo el grupo, había visto formalmente sólo un curso de mecánica, en su física de grado décimo (penúltimo año de bachillerato); excepto dos alumnos que ya habían cursado un semestre en otra carrera, en la cual como parte del plan de estudios vieron física.

\section{B. El curso y su análisis categorial}

Ya nos hemos referido anteriormente a que tanto los programas de estudio (de bachillerato y de universidad), como casi la totalidad de textos la práctica usual, inician el estudio de la física por la cinemática. Pero trabajos previos como el de (Ortiz, 1986), proponen iniciar con los fenómenos relacionados con la Dinámica por cuanto la tendencia inicial de los alumnos es dar cuenta de las causas del movimiento y no describirlo cinemática-. Para casi todos la descripción es inseparable de la consideración de las 
causas y circunstancias en que se da el movimiento, existe entonces una negativa sistemática a considerar el movimiento independientemente de las causas.

Una vez elegido el tema de fuerza y movimiento y antes de iniciar con las lecturas sobre los pensamientos de Aristóteles a Newton, iniciamos el curso con la construcción por parte del grupo, a partir de la búsqueda bibliográfica individual, de un panorama general de las cuatro grandes épocas en que se ha dividido la historia de la humanidad (ver cuadro $\mathrm{N}^{0} 1$ ): esto con el fin de que los alumnos ubicaran algunos de los personajes más representativos de cada período, inventos, descubrimientos, ternas de investigación, preocupaciones, aportes y características de cada una de ellas, en el campo de la ciencia; máxime cuando algunos de estos elementos les han sido enseñados en sus cursos de ciencias de bachillerato. Además, elaboraron un trabajo escrito sobre el movimiento" y las clases de movimiento, de acuerdo a sus propios criterios, pues para la realización de dicho trabajo sólo se presentaron a los estudiantes algunos lineamientos generales.

Cuadro 1. Las cuatro grandes épocas de la humanidad.

\begin{tabular}{|c|c|c|c|}
\hline $\begin{array}{l}\text { EDAD ANTIGUA } \\
\text { A.C. - V D.C. }\end{array}$ & $\begin{array}{c}\text { EDAD MEDIA } \\
\text { V-IX }\end{array}$ & $\begin{array}{c}\text { EDAD MODERNA } \\
\text { XV-XVIII }\end{array}$ & $\begin{array}{l}\text { CONTEMPORANEA } \\
\text { XVIII-XX }\end{array}$ \\
\hline $\begin{array}{l}\text { Mov. Terrestres } \\
\text { Mov. Celestes }\end{array}$ & $\begin{array}{c}\text { Iglesia } \\
\text { Teoría geocéntrica }\end{array}$ & $\begin{array}{l}\text { Rev. Copernicana } \\
\text { Rev. Galileana } \\
\text { Teoría Heliocéntrica }\end{array}$ & $\begin{array}{l}\text { Rev. Einsteniana } \\
\text { Mundo microscópico }\end{array}$ \\
\hline & Oscurantismo & Renacimiento & $\begin{array}{l}\text { Especialización de } \\
\text { Las ciencias }\end{array}$ \\
\hline $\begin{array}{c}\text { Aristóteles, Pitágoras } \\
\text { Platón }\end{array}$ & $\begin{array}{l}\text { Oresme } \\
\text { Arquímedes }\end{array}$ & $\begin{array}{c}\text { El hombre es el centro del universo } \\
\text { como ser pensante } \\
\text { Rev. Newtonian } \\
\text { (las tres leyes de Newton) } \\
\text { Cavendish } \\
\text { Maxwell }\end{array}$ & $\begin{array}{l}\text { Plank, Bohr, Thompson } \\
\text { Madame Curie }\end{array}$ \\
\hline
\end{tabular}

Lo anterior, especialmente con la intención de que luego ellos mismos vieran por ejemplo, cómo recitaban muy bien la teoría heliocéntrica y las tres leyes de Newton que aprendieron en $1 \mathrm{Q} \sim$ grado, pero a la hora de dar respuesta a una pregunta o explicar un fenómeno, su pensamiento parecía seguir siendo de la antigüedad.

Algunos de los aspectos más importantes que se incluyeron en las actividades que se realizaron pretendía que los estudiantes verbalizaran sus concepciones respecto a las diferentes preguntas o situaciones planteadas. Para lo cual podríamos decir que el guión que se siguió fue generalmente lectura previa, discusión en pequeños grupos y plenaria ó planteamiento de una situación problemática al grupo en pleno, discusión, análisis y luego lectura complementaria.

Nuestro texto de lectura o texto guía tiene la particularidad en primer lugar de hacer poco uso de las matemáticas, lo cual nos aleja de los típicos textos de fórmulas y además, contiene citas anecdóticas, fotografías y poemas, resúmenes biográficos y consideraciones históricas, que se propone ser un libro de física y también sobre física; pero ante todo es un libro que expone las ideas de la física tal como evolucionaron, proporcionando así una buena panorámica a los estudiantes de a naturaleza propia de la física. 
Dentro de esta perspectiva se trabajaron los tres primeros capítulos del libro de Hecht, acompañados como ya lo dijimos anteriormente de videos, experiencias sencillas en clase y debates entre los estudiantes, tanto en pequeños grupos como en plenaria, respecto a sus concepciones y respecto al cambio mismo de las ideas a lo largo de la historia. También es necesario mencionar que incluso como parte del curso se leyó el libro Galileo Galilei de Bertold Bretch.

A continuación presentaremos cuatro ejemplos a partir de preguntas o situaciones problémicas, del trabajo de campo, las cuales consideramos fueron las más significativas en torno al cambio de paradigma y elaboraremos un breve ana-lisis de cada una de ellas.

Cuatro Preguntas y un Breve Análisis. En el cuestionario final que se realizó dos meses después de terminado el curso, se solicitó a los alumnos que hicieran un paralelo entre la concepción Aristotélica y la Newtoniana, y que plantearan 4 preguntas respecto a los conceptos abordados a lo largo del curso, que más hubieran sido para ellos significativos y que le dieran respuesta a estas preguntas. Nos pareció muy sugerente la coincidencia en las preguntas libremente planteadas, razón perla cual berros seleccionado algunas de ellas; las cuales corresponden a nuestros tres últimos ejemplos.

\section{Un mundo geocéntrico dentro de un universo heliocéntrico}

Se planteó a los estudiantes la siguiente situación: Si usted se encuentra en un lugar perdido y sin ayuda de brújula o cualquier otro tipo de instrumento, pero sabe que si camina hacia el oriente encontrará el camino de regreso a casa, ¿cómo haría para saber cuál es el oriente y cuál el occidente?

La respuesta casi inmediata y al unísono fue por donde sale e/soles el oriente y por donde se oculta ese/occidente. Enseguida leyeron la concepción del universo aristotélico y se sorprendieron al ver que ellos también ponen en movimiento al sol y dejan a la tierra en reposo. Lo cual les permitió reflexionar y concluir que: aprobarnos el curso de física de $10^{\circ}$, pero no cambiaron nuestras ideas.

Después de seguir la pista a las fallas o anomalías de la teoría geocéntrica respecto de la observación experimental, a las hipótesis propuestas por Ptolomeo hasta llegan a la propuesta copernicana, a través de la lectura y de los videos, los alumnos decían: ya entendemos porque Aristóteles estaba equivocado. Aunque ellos parecen entender la causa por la cual el paradigma copernicano reemplazó al milenario paradigma aristotélico les causa dificultad dar respuesta a la pregunta inicial desde este nuevo referente teórico, pues aparentemente va en contra de su experiencia cotidiana.

\section{Concepción indiferenciada de gravedad, fuerza gravitacional y peso}

Al inicio de una sesión de clase se lanzó la siguiente pregunta: ¿si se sostiene un cuerpo con las manos y luego se deja de sujetar, por qué cae el cuerpo? A lo cual una parte del grupo respondió por la acción de la gravedad y ¿que es la gravedad? - la gravedades una fuerza igual que actúa sobre todos los cuerpos y vale $9,8 \mathrm{~m} / \mathrm{s}^{2}$ otros dijeron que: los cuerpos tienden a caer debido al peso que poseen y, ¿qué es el peso? Es masa por gravedad. Lo anterior permite identificar varias cosas: si bien manejan cierta información como el valor de la gravedad y la expresión matemática para calculan el peso, no saben conceptualtzar claramente cada uno de ellos; hay confusión entre estos conceptos, lo cual no les permite diferenciar y establecer las correspondientes relaciones entre la gravedad como aceleración, la fuerza gravitacional y el peso. Además, cuando los 
alumnos dicen que los cuerpos tienden se deja entrever en su respuesta una explicación animista de los fenómenos.

Luego se les cuestionó sobre cuáles serían las respuestas de Aristóteles y Newton a la pregunta: ¿por qué cae una manzana? - Interrogante que propició inicialmente un gran silencio en el salón- luego l'inieron respuestas como uhmm, bueno Aristóteles diría que porla composición de la manzana, ya que tiene mas cercanía al elemento tierra, que a los otros tres, no, respondería que es por que los cuerpos tienden a ir a su estado natural,es decir, a caer, y respecto a la perspectiva newtoniana: diría que es debido a la acción de la gravedad, otros, sí, debido a que la fuerza de gravedad que hace que todos los cuerpos caigan hacia la tierra.

Resultó muy interesante cuando los alumnos vieron que sus respuestas a la primera situación problemática eran similares a las que ellos dieron cuando se les cuestionó sobre los argumentos de Aristóteles y de Newton, pues los hizo reflexionar sobre la solidez de sus respuestas.

\section{De la fuerza a la fuerza neta}

Luego de abordar el capitulo referente a las leyes de Newton se preguntó a los alumnos en uno de los cuestionarios si ¿Cuándo un cuerpo está en movimiento con velocidad constante actúa alguna tuerza sobre él'?,- la respuesta predominante fue: No, sobre el cuerpo no actúa ninguna fuerza. Otros contestaron que: actúa una fuerza constante sobre él.

Lo que nos llevó a revisar en detalle la construcción de las leyes de Newton, pues si bien la fuerza neta externa es cero, esto significa que no actúe ninguna fuerza sobre el cuerpo; justamente lo que sucede es que dichas fuerzas se anulan; lo que permite que el cuerpo se mueva con velocidad constante. Así pues fue necesario pasar el concepto de fuerza (como acción unitaria) de tipo aristotélico al concepto de fuerza neta, al estilo newtoniano.

En una clase se planteó una situación de un cuerpo que bajaba acelerado por un plano inclinado sin rozamiento y se solicitó a los alumnos que ubicaran las fuerzas que actuaban sobre el cuerpo; la gran mayoría estuvo de acuerdo con tres fuerzas: una en dirección vertical hacia abajo que denominaron el peso, otra perpendicular saliendo del plano; es decir, la normal y una tercera que iba hacia abajo, paralela al plano inclinado. Así, aunque reconocen la presencia de varias fuerzas, persisten en la idea de que debe actuar directamente sobre e! cuerpo una fuerza que vaya en la misma dirección del movimiento y no que sea la de la dirección de la fuerza neta.

\section{Se le acabo la fuerza}

Se solicitó a los estudiantes que explicaran por qué después de cierto tiempo de observar un balón moviéndose horizontalmente sobre el suelo éste se detenía. Aproximadamente la mitad del curso dijo: se le acabó la fuerza de empuje, la otra mitad argumentó que se le acabo el impulso... es más, entre mayor sea el impulso, mas se tardará en detenerse: y algunos pocos mencionaron que se debe al rozamiento: pero al preguntarles qué era el rozamiento decían cosas como es lo que distingue a uno 
superficie lisa de una rugosa o áspera. Pero no podían precisar si era una característica propia de la superficie llamada coeficiente de rozamiento, o si correspondía a una tuerza.

Las repuestas dadas por los alumnos nos remiten a la explicación medieval del ímpetu. El cual permite al cuerpo continuar en movimiento aun después de haber abandonado el contacto con el cuerpo que lo impulsó y parece que va desapareciendo a medida que se usa y la respuesta, deque para que haya movimiento se requiere de una fuerza fue una constante que se ratifico en diferentes situaciones con expresiones como: paro continuar moviéndose a la velocidad constante, hace taita un impulso constante es decir; sirio hay fuerza, no hay movimiento, lo cual nos recuerda la teoría aristotélica

Como la situación antes mencionada fue una de las primeras del semestre, ésta dio pie para dirigir nuestra lectura y actividades experimentales con el fin de dar cuenta sobre la evolución de la relación entre fuerza y movimiento hasta llegar a la presentarla por la física escolar.

Al final del curso los estudiantes realizaron un paralelo entre la concepción aristotélica y newtoniana del movimiento; he aquí algunos ejemplos:

Aristóteles afirmaba que sin una fuerza impulsora no sería posible el roo cimiento; se basaba solo en la observación y Newton afirmaba que la fuerza actúa sobre un cuerpo para producir un cambio en el movimiento.

Aristóteles decía que los cuerpos se mantenían en movimiento porque el aire era un fuerza que los empujaba y Newton decía que los cuerpos se movían porque se alteraba su inercia o su estado de equilibrio. Según Aristóteles, para que cuerpo mantuviera su movimiento era necesario que una tuerza impulsora actuara constante sobre el, y según Newton, para que un cuerpo mantuviera su movimiento era necesario que la sumatoria de fuerzas fuera igual a cero.

Así pues, de alguna manera se superó el credo de las tres leyes respecto a la concepción newtoniana al buscar que ellos identificaran la inconmensurabilidad entre el paradigma aristotélico y ei newtoniano, teniendo como referente situaciones particulares.

\section{Otros resultados}

A partir de la implementación didáctica de la historia de la ciencia como herramientaa para la construcción de significados en el curso de física, vale la pena destacar que los alumnos de la clase de física montaron en versión moderna, la obra de teatro Galileo Galilei de Bertold Bretch; la cual fue presentada ante toda la Facultad de Ciencias, pretendiendo mostrar por un lado la relevancia de los aportes de Galileo a la Física y por otro, la importancia de la historia interna y externa de las ciencias en la construcción del conocimiento.

\section{Análisis de los actores que intervienen}

Dado que la investigación que se llevó a cabo fue de tipo etnográfica - cualitativa, basada en el análisis de una experiencia particular; estimamos importante transcribir algunas consideraciones y percepciones, sobre este tipo de trabajos, por parte de los alumnos que participaron en esta experiencia: 
- El método de conocerla vida e ideas de las primera personas que se detuvieron a observar el movimiento, como la Tierra ocupaba un lugar en el Universo y en qué lugar es bueno, ya que uno sale de errores y también certifica algunas buenas ideas que se tienen; por otro lado es correcto conocer algunos de los primeros sabios que existieron en la historia.

- La diferencia con otras clases es notable, ya que se lee más y uno se entera de dónde han salido estas ideas.

- El curso no se trató de que aprendiéramos fórmulas y conceptos, como se hace en otras clases, sino que ante todo de conocerlas raíces y pensamientos antiguos de los más célebres físicos, sus experimentos y conclusiones, para llegar a lo que hoy conocemos acerca de la física.

- La clase no fue conocer fórmulas matemáticas. Pudimos expresar nuestros pensamientos. Pues generalmente a uno como alumno no lo tienen en cuenta en la Universidad, somos uno más del montón.

Los argumentos expuestos por los estudiantes a favor de que se consideren sus propios puntos de vista y de ser reconocidos como parte importante de la acción didáctica, no como cerebros vírgenes o tablas rasas, constituye un importante aporte y un elemento de reflexión, que permite validar nuestra hipótesis sobre las implicaciones del enfoque histórico para la construcción de significados en las clases de física.

\section{CONCLUSIONES}

A continuación reseñamos algunas reflexiones a modo de conclusiones generales sobre los cuatro ejemplos, que encierran el núcleo central de nuestro trabajo, frente al problema de fuerza y movimiento:

- Puede ocurrir que sobre un cuerpo actúen varias fuerzas, pero si la fuerza neta es cero, se moverá como si no actuara sobre él ninguna fuerza. Esta es una situación más realista, pues nunca se tiene un cuerpo verdaderamente libre de fuerzas.

- Según Aristóteles, sólo el estado de reposo era perdurable; para que un cuerpo se mueva es necesario aplicar constantemente una fuerza; de manera contraria Newton consideró la fuerza como un agente de cambio, es decir, la fuerza para Newton es causa del cambio en la cantidad de movimiento que es una cantidad constante igual o diferente de cero-reposo o movimiento uniforme, respectivamente- en cambio para Aristóteles la fuerza es la causa del movimiento.

- Las explicaciones animistas, como los cuerpos tienden, son provocadas e inducidas por los mismos libros de texto, por ejemplo cuando se enuncia la primera ley de Newton, encontramos definición corno: Los cuerpos tienden a permanecer en su estado de reposo o movimiento rectilíneo uniforme...

Puesto que el problema de la enseñanza y el aprendizaje de la ciencia se ha constituido en uno de los retos más significativos para la didáctica de las ciencias, al tomar partido por la perspectiva histórica y de acuerdo con la hipótesis y los objetivos planteados en el primer capitulo, podemos concluir que: 
El trabajo de investigación que aquí se reporta muestra similitudes con otras investigaciones que se encuentran en la literatura anglosajona, desarrolladas en otros contextos culturales alrededor de las ideas previas de los alumnos referentes al problema de tuerza y movimiento descrito en los cuatro ejemplos presentados, pero además se consolida como un aporte dentro de la investigación sobre la enseñanza de la s ciencias experimentales entorno al enfoque histórico y las ideas porevias, puesto que reporta una experiencia particular de aula, llevada a cabo a lo largo de todo un semestre por un profesor investigador y no se queda en el único plano de los análisis o propuestas de tipo teórico.

De acuerdo fundamentalmente con el análisis de los actores que intervienen, los alumnos logran identificar y establecer correspondencias o semejanzas entre sus ideas explicativas con las proferidas por los hombres de ciencia en algún momento de la historia de la física, lo cual les parece fascinante y se convierte en un elemento motivacional para el aprendizaje, corno cambio conceptual. Lo que resulta muy significativo máxime cuando los alumnos provienen de disciplinas diferentes a la física, corno en este caso, alumnos de biología y química, permitiendo superar así un poco la visión de ladrillo que se tiene de la física.

- Finalmente vamos a permitirnos resaltar aquí algunos aspectos a manera de sugerencias que para el caso son importantes y corresponden al ámbito general de la enseñanza:

- Hay que tener en cuenta que la rigidez del currículo y del sistema dificulta el desarrollo de la propuesta, pues este tipo de trabajo exige la participación del estudiante el cual está acostumbrado generalmente a que la clase sea expositiva y a la memorización. De tal manera que no resulta sencillo que verbalicen y sustenten sus ideas tan claramente corno uno puede pensar, cambiar de paradigma no es fácil.

- La perspectiva epistemológica del profesor de ciencias juega un papel importante en el proceso enseñanza-aprendizaje, pues compartimos la hipótesis que los compromisos epistemológicos de los docentes o sus concepciones sobre la ciencia inciden en el aula de clase y tienen efectos directos sobre sus alumnos es decir, los resultados de una estrategia didáctica como el enfoque histórico están mediados inevitablemente por la concepción epistemológica del maestro.

- Respecto a la escasez del material didáctico tanto escrito como experimental que pueda apoyar cursos de física desde este enfoque, quedan abiertas las puertas para realizar trabajos que busquen llenar este vacío.

- Ante la resistencia por parte de los estudiantes para transformar sus ideas previas sería recomendable recurrir a experiencias sencillas que por lo menos generen la posibilidad de la duda respecto a las observaciones del sentido común, como el ejemplo de la manzana que propropone Galileo a Andrea, en la obra de Bertold Bretch, y no recurrir a explicaciones o situaciones experimentales complejas que son difíciles de llevar a cabo en el aula, como la del péndulo de Foucault.

\section{BIBLIOGRAFÍA}

Base Duit (1998) Bibliography en students alternative frameworks and science education. 
Carey, S, (1992). The original evolution of everyday concepts. En R. Giere (Ed.), Cognitive Models of Science. Minnesota Studies in the Philosophy of Science. Volume XV (pp. 89128). University of Minnesota Press.

Champagne. B. Gunstone, F. \& Klopfer, E. (1985). Instructional consequences of students. knowledge about physical phenomena. En Leo, West \& Pinos (Eds.), Cognitive Structure and Conceptual Change (pp.61 -90). Academic Press.

Clement. J. (1983). A conceptual model discussed by Galileo and used intuitively by physcs students. En Gentener \& Stevens (Eds.), Mental Models (pp. 325-339). Lawrence Erlbaum Associates.

Driver, H. \& Easley. J. (1978). Pupils and panadigms: a review of literature related to concept development in adolescent science students. Studies in Science Education, II, $61-84$.

Fischbein E Stavy, R \& Ma-Haim, H. (1989). The psychological structure of naive impetus conceptions. International Journal of Science Education, 11(1), 71-81.

Fund, C. \& Guisasola J. (1999). Concepciones alternativas y dificultades de aprendizaje en electrostática. Selección de cuestiones elaboradas para su detección y tratamiento. Enseñanza de las Ciencias, 17(3 ). 441 -452.

Gagliard, R. (1988). Cómo utilizar la historia de las ciencias en la enseñanza de las ciencias. Enseñanza de las Ciencias, 4 t3), 291-296.

García. 0. (1998). La Historia del Desarrollo del Pensamiento Científico. Manizales: Universidad de Manizales, Colombia.

Gil. D. (1993). Contribuciones de la historia y la filosofía de las ciencias al desarrollo de un modelo de enseñanza / aprendizaje como investigación, Enseñanza de las Ciencias, 11(2), 197- 212.

Gunstone R. F. \& Watts, D.M (1985). Force and Motion. En R. Driver, E. Guesne \& A. Tiberghien (Eds.), Children's ideas o science (pp. 84-104). Milton Keynes, Philadelphia: Open University Press.

Hammer. D. (1994). Epistemological beliefs in introductory Physics, Cognition and instruction, 12, 151-183.

Hecht, E. (1987). Física en Perspectiva. Massachusetts, Addison Wesley lberoamericana. SA.

Hewson, P. W. (1990). La enseñanza de la fuerza y el movimiento como cambio conceptual. Enseñanza de las Ciencias. 8(2), 157-171.

Khun, T. S. (1984). Estructuras de las Revoluciones Científicas. México, Fondo de Cultura Económica.

Matthews, M. (1990). History, philosophyand science teaching: a rapprochement, Studies in Science Education, 18, 25-51. 
McCloskey. M. (1983). Naive theories of motion, En Gentener \& Stevens (Eds). Mental Models (pp. 299-324) Lawrence Erlbaum Associates.

Newton, I. (1994) Principios matemáticos de la Filosofía natural. Barcelona, Ediciones Altaya.

Ortiz, D. (1986) Detección de dificultades en la enseñanza del concepto de movimiento a nivel medio. Tesis de maestría no publicada, Universidad Pedagógica Nacional, Bogotá D, E., Colombia.

Osborne, R. \& Witrock, M. (1983), Learning science: A generative process, Science Education, 67, 489-508.

Peduzzi, L \& Zylbersztajn, A. (1997). La física de la fuerza impresa y sus implicaciones para la enseñanza de la mecánica, Enseñanza de las Ciencias, 15(3), 351-359.

Pérez Ransanz, AB. (1999). Kuhn y e ICambio Científico. México, Fondo de Cultura Económica.

Pessoa de Carvalho, AM. \& Castro, RS (1992) La historia de la ciencia como herramienta para la enseñanza de la física en secundaria: un ejemplo en calor y temperatura, Enseñanza de las Ciencias, 10(3). 289-294

Pfundt, H. \& Ouit, R. (1998) Bibhography on students alternative frameworks and sciencie educafion, Kiel, Alemania: Institut für Piidagogik der Naturwissenschaften

Solano. I. Jiménez, E. \& Marín. N. (2000). Análisis de la metodología utilizada en la búsqueda de lo que el alumno sabe sobre fuerza, Enseñanza de las Ciencias, 18(21, 171-188.

Viennot, L, (1979). Spontaneous reasoning in elementary dynamics.. En European Journal of Science Educafion, 1(2), 205-221.

Wandersee, J., Mintzes, J. \& Novak, J. (1994). Research on alternative conceptions in science, En D. Gabel (ed). Handbook of Research en Science Teaching and Learning MacMillan Pub.: New York.

Whitaker, R. J (1983). Aristotle is not dead: student understanding of trajectory motion. En American Journal of Physics, 51, 352-357 